\title{
Hybrid TOA/AOA Approximate Maximum Likelihood Mobile Localization
}

\author{
Mohamed Zhaounia, ${ }^{1}$ Mohamed Adnan Landolsi, ${ }^{2}$ and Ridha Bouallegue ${ }^{1}$ \\ ${ }^{1}$ Unité de recherche 6’Tel, Ecole Superieure des Communications, El Ghazala, Ariana 2083, Tunisia \\ ${ }^{2}$ EE Department, King Fahd University, Dhahran 31261, Saudi Arabia
}

Correspondence should be addressed to Mohamed Zhaounia, mzhaounia@yahoo.fr

Received 9 June 2009; Accepted 3 November 2009

Academic Editor: Montse Najar

Copyright ( $\odot 2010$ Mohamed Zhaounia et al. This is an open access article distributed under the Creative Commons Attribution License, which permits unrestricted use, distribution, and reproduction in any medium, provided the original work is properly cited.

This letter deals with a hybrid time-of-arrival/angle-of-arrival (TOA/AOA) approximate maximum likelihood (AML) wireless location algorithm. Thanks to the use of both TOA/AOA measurements, the proposed technique can rely on two base stations (BS) only and achieves better performance compared to the original approximate maximum likelihood (AML) method. The use of two BSs is an important advantage in wireless cellular communication systems because it avoids hearability problems and reduces network signaling burden. Simulation results show that, for certain scenarios, the proposed hybrid TOA/AOA AML with two BSs can outperform the AML with up to six BSs.

\section{Introduction}

Mobile positioning in wireless communication has gained a wide acceptance and trigged a large number of innovative applications [1,2]. Various techniques have been proposed for mobile positioning, depending on the collected measurements by the wireless networking infrastructure. Broadly, these techniques can be classified as (i) time-based location that produces a circle centered at the base station (BS) and the mobile station (MS) location estimate is determined by the intersection of three circles at least, and (ii) angle-based location, which requires BSs equipped with antenna arrays, defines a line of bearing from the BS to the MS whose position is calculated from the intersection of a minimum of two BSs.

Because of the noisy measurements, the mobile position is typically determined by some statistical estimation which processes a set of nonlinear equations constructed from the TOA and/or AOA statistics with knowledge of the BSs geometry. Among the different techniques proposed, one particularly interesting approach known as approximate maximum likelihood (AML) was developed in [3] and shown to achieve near-optimal performance without the complexity of "full" maximum likelihood estimation. One limitation of the original AML method in [3] is that it relies on TOA data only and needs measurements from several BSs (all assumed to have the same measurement error variance). However due to hearability problem, the signal received at BSs other than the serving BS may not be strong enough to provide reliable measurements [4]. Furthermore, the use of a large number of BSs results in a network overload by requiring complex messaging and synchronization. As such, obtaining reliable measurements from at least three BSs becomes difficult to achieve in real-world scenarios. In this letter, we present a new "hybrid" version of the AML algorithm by incorporating both TOA and AOA data and show that this new positioning method can have superior performance by using measurements from only 2 BSs.

\section{The AML Approach}

This section gives a brief description of the original AML approach in [3]. Assuming $N \geq 3$ BSs involved in the positioning process, the vector of measured distances is

$$
\Delta=\left[\begin{array}{lll}
\delta_{1} & \cdots & \delta_{N}
\end{array}\right]^{T},
$$


where $\delta_{i}=c t_{i}$ with $t_{i}$ being the measured TOA of the $i$ th BS and $c$ stands for the light velocity.

The measured TOA data are assumed to be corrupted by independent zero-mean Gaussian noise with a covariance matrix given by

$$
\mathbf{Q}=\operatorname{diag}\left\{\operatorname{var}_{\mathrm{TOA}_{1}} \cdots \operatorname{var}_{\mathrm{TOA}_{N}}\right\},
$$

where the variances $\left(\operatorname{var}_{\mathrm{TOA}_{i}}\right)_{1 \leq i \leq N}$ are not necessarily equals.

The calculated vector $\mathbf{R}$ is obtained from the estimated vector $\theta=\left[\begin{array}{l}x y \\ ]^{T}\end{array}\right.$

$$
\mathbf{R}(\theta)=\left[\begin{array}{lll}
r_{1} & \cdots & r_{N}
\end{array}\right]^{T}
$$

where

$$
r_{i}^{2}=s+k_{i}-2 x x_{i}-2 y y_{i}
$$

with $\left(x_{i}, y_{i}\right)$ being the coordinates of the $i$ th BS and

$$
\begin{aligned}
& s=x^{2}+y^{2}, \\
& k_{i}=x_{i}^{2}+y_{i}^{2} .
\end{aligned}
$$

Then, the probability density function (pdf) [5] of $\Delta$ given $\theta$ is expressed as

$$
f(\Delta \mid \theta)=(2 \pi)^{-N / 2} \operatorname{det}(\mathbf{Q})^{-1 / 2} \exp \left\{-\frac{J}{2}\right\},
$$

where

$$
J=(\Delta-\mathbf{R}(\theta))^{T} \mathbf{Q}^{-1}(\Delta-\mathbf{R}(\theta)) .
$$

The ML estimation principle seeks to maximize $f(\Delta \mid \theta)$. Then, setting the gradient of $J$ with respect to $\theta$ to zero gives two nonlinear ML equations, which are treated iteratively in a linear way in the simplified AML approach of [3]. Starting from some initial position, it first computes the coefficients of those equations expressed as a function of $\theta$. Then applying (4) produces a quadratic in $s$. Next, a root selection routine (RSR) chooses the correct root. Finally, the AML selects the $\theta$ that gives the minimum $J$.

\section{The Proposed HAML Approach}

In the proposed approach the serving BS provides both the AOA and TOA measurements. By introducing the AOA information, we use statistics from two BSs only. Then, the vector of measured parameters becomes

$$
\Delta_{H}=\left[\begin{array}{lll}
\delta_{1} & \delta_{2} & \beta
\end{array}\right]^{T},
$$

where $\beta$ is the AOA at the home BS corrupted by an additive zero-mean Gaussian noise with variance $\operatorname{var}_{\mathrm{AOA}}$ independent from TOA noise. The covariance matrix becomes

$$
\mathbf{Q}_{H}=\operatorname{diag}\left\{\operatorname{var}_{\mathrm{TOA}_{1}} \quad \operatorname{var}_{\mathrm{TOA}_{2}} \quad \operatorname{var}_{\mathrm{AOA}}\right\} .
$$

In the same way, the calculated vector includes the AOA measurement and is obtained as

$$
\mathbf{R}_{H}(\theta)=\left[\begin{array}{lll}
r_{1} & r_{2} & \alpha
\end{array}\right]^{T}
$$

where $\alpha=\arctan (y / x)$ and, without loss of generality, the serving BS is assumed at the origin (0.0). Then, the pdf of $\Delta_{H}$ given $\theta$ is given by

$$
f\left(\Delta_{H} \mid \theta\right)=(2 \pi)^{-3 / 2} \operatorname{det}\left(\mathbf{Q}_{H}\right)^{-1 / 2} \exp \left\{-\frac{J_{H}}{2}\right\},
$$

where $J_{H}$ is expressed as

$$
J_{H}=\sum_{i=1}^{2} \frac{\left(\delta_{i}-r_{i}\right)^{2}}{\operatorname{var}_{\mathrm{TOA}_{i}}}+\frac{(\beta-\alpha)^{2}}{\operatorname{var}_{\mathrm{AOA}}} .
$$

Thus, the ML estimate is the vector $\theta$ that minimizes $J_{H}$.

Setting the gradient of $J_{H}$ with respect to $\theta$ to zero yields the following system of equations:

$$
\begin{aligned}
& \sum_{i=1}^{2} \frac{\left(x-x_{i}\right)\left(r_{i}-\delta_{i}\right)}{\operatorname{var}_{\mathrm{TOA}} r_{i}}+\frac{\left(y-y_{1}\right)(\beta-\alpha)}{\operatorname{var}_{\mathrm{AOA}} r_{1}^{2}}=0, \\
& \sum_{i=1}^{2} \frac{\left(y-y_{i}\right)\left(r_{i}-\delta_{i}\right)}{\operatorname{var}_{\mathrm{TOA}_{i}} r_{i}}-\frac{\left(x-x_{1}\right)(\beta-\alpha)}{\operatorname{var}_{\mathrm{AOA}} r_{1}^{2}}=0 .
\end{aligned}
$$

Substituting the quantity $r_{i}-\delta_{i}=\left(r_{i}^{2}-\delta_{i}^{2}\right) /\left(r_{i}+\delta_{i}\right)$, the system of equations above becomes

$$
\begin{aligned}
& \sum_{i=1}^{2} p_{i}\left(s+k_{i}-\delta_{i}^{2}\right)=\sum_{i=1}^{2} p_{i}\left(2 x_{i} x+2 y_{i} y\right)-2 w\left(y-y_{1}\right) \\
& \sum_{i=1}^{2} q_{i}\left(s+k_{i}-\delta_{i}^{2}\right)=\sum_{i=1}^{2} q_{i}\left(2 x_{i} x+2 y_{i} y\right)+2 w\left(x-x_{1}\right)
\end{aligned}
$$

where $p_{i}=\left(x-x_{i}\right) /\left(\operatorname{var}_{\mathrm{TOA}_{i}}\left(r_{i}+\delta_{i}\right)\right), q_{i}=\left(y-y_{i}\right) /$ $\left(\operatorname{var}_{\mathrm{TOA}_{i}}\left(r_{i}+\delta_{i}\right)\right)$ and $w=1 / 2(\beta-\alpha) /\left(\operatorname{var}_{\mathrm{AOA}} r_{1}^{2}\right)$.

Then in matrix notation

$$
2 \mathbf{A} \theta=\mathbf{b}_{1} s+\mathbf{b}_{2}
$$

where

$$
\begin{gathered}
\mathbf{A}=\left[\begin{array}{cc}
\sum_{i=1}^{2} p_{i} x_{i} & \sum_{i=1}^{2} p_{i} y_{i}-w \\
\sum_{i=1}^{2} q_{i} x_{i}+w & \sum_{i=1}^{2} q_{i} y_{i}
\end{array}\right], \\
\mathbf{b}_{1}=\left[\begin{array}{c}
\sum_{i=1}^{2} p_{i} \\
\sum_{i=1}^{2} q_{i}
\end{array}\right], \quad \mathbf{b}_{2}=\left[\begin{array}{l}
\sum_{i=1}^{2} p_{i}\left(k_{i}-\delta_{i}^{2}\right)-2 w y_{1} \\
\sum_{i=1}^{2} q_{i}\left(k_{i}-\delta_{i}^{2}\right)+2 w x_{1}
\end{array}\right] .
\end{gathered}
$$

Performing the Least Squares (LS) estimator [5] leads to

$$
\begin{gathered}
x=\theta(1)=\frac{1}{2}\left(\mathbf{A}^{-1} \cdot \mathbf{b}_{1}\right)(1) s+\frac{1}{2}\left(\mathbf{A}^{-1} \cdot \mathbf{b}_{2}\right)(1)=a s+b, \\
y=\theta(2)=\frac{1}{2}\left(\mathbf{A}^{-1} \cdot \mathbf{b}_{1}\right)(2) s+\frac{1}{2}\left(\mathbf{A}^{-1} \cdot \mathbf{b}_{2}\right)(2)=a^{\prime} s+b^{\prime} .
\end{gathered}
$$


Substituting (18) into (5) a quadratic in $s$ is obtained:

$$
(a s+b)^{2}+\left(a^{\prime} s+b^{\prime}\right)^{2}=s .
$$

Solving (19) raises 3 possible cases.

Case 1. The roots are real and there is only one positive root. Then $s$ takes the value of this positive root and $\theta$ is estimated by applying (18).

Case 2. The roots are real and are both positive. Then $\theta$ is calculated by applying (18) for each root and the corresponding value of $J_{H}$ is determined by performing (13). The correct root is the one that leads to the minimum value of $J_{H}$ and the estimated $(x, y)$ is the corresponding $\theta$.

Case 3. The roots are negative (or complex). In this case we take the absolute value (of the real part) and process as in the Case 2.

In order to calculate the initial estimate, we start with the noise-free case,

$$
\begin{gathered}
s+k_{i}-\delta_{i}^{2}=2\left(x x_{i}+y y_{i}\right), \\
\left(x-x_{1}\right) \sin \beta-\left(y-y_{1}\right) \cos \beta=0 .
\end{gathered}
$$

In matrix notation, this is equivalent to

$$
\mathbf{B} \theta=\frac{1}{2} \mathbf{c}_{1} s+\mathbf{c}_{2},
$$

where

$$
\begin{gathered}
\mathbf{B}=\left[\begin{array}{cc}
x_{1} & y_{1} \\
x_{2} & y_{2} \\
\sin \beta & -\cos \beta
\end{array}\right], \\
\mathbf{c}_{1}=\left[\begin{array}{l}
1 \\
1 \\
0
\end{array}\right], \quad \mathbf{c}_{2}=\left[\begin{array}{c}
\frac{1}{2}\left(k_{1}-\delta_{1}^{2}\right) \\
\frac{1}{2}\left(k_{2}-\delta_{2}^{2}\right) \\
x_{1} \sin \beta-y_{1} \cos \beta
\end{array}\right] .
\end{gathered}
$$

An initial position can be obtained from the LS estimate as

$$
\theta=\frac{1}{2}\left(\mathbf{B}^{T} \mathbf{B}\right)^{-1} \mathbf{B}^{T}\left\{\mathbf{c}_{1} s+\mathbf{c}_{2}\right\} .
$$

Combining (23) and (5) provides a quadratic in $s$. Then, the adequate root is chosen similarly as discussed in (19).

As can be seen and since it uses only 2 BSs, the proposed HAML algorithm is applicable regardless of the BSs' geometry. In fact unlike the original AML algorithm, the HAML does not discriminate between cases where the BSs are on a straight line or not, which can be considered as an important advantage of the HAML regarding the implementation complexity of the AML in the case of 3 linear BSs [3].
TABLE 1: Performance comparison of 2-BS HAML and AML with various number of BSs.

\begin{tabular}{lcccccc}
\hline \multicolumn{3}{c}{ HAML } & \multicolumn{5}{c}{ AML } \\
\hline \# BS & 2 BSs & 3 BSs & 4 BSs & 5 BSs & 6 BSs & 7 BSs \\
RMS (Km) & 0.0944 & 0.1117 & 0.1019 & 0.0991 & 0.0972 & 0.0937 \\
\hline
\end{tabular}

\section{Numerical Results}

This section presents simulation results that illustrate the performance improvement of the HAML approach using 2 BSs only, compared to the original AML method using a larger number of BSs.

In these simulations a hexagonal wireless cellular system layout with a radius $R_{c}=4 \mathrm{Km}$ is considered. TOA and AOA measurements are assumed to be corrupted by independent Gaussian noise with variances $\operatorname{var}_{\mathrm{TOA}}$ and $\operatorname{var}_{\mathrm{AOA}}$, respectively. Due to range differences and geometrical distribution, we assume that the TOA standard deviation of the home BS is set to be $50 \mathrm{~m}$ and increases linearly with a step of $50 \mathrm{~m}$ for the other BSs without exceeding $200 \mathrm{~m}$, that is, $100 \mathrm{~m}$ for the second BS, $150 \mathrm{~m}$ for the third BS, and $200 \mathrm{~m}$ for the other BSs. The AOA standard deviation is fixed to be $3^{\circ}$. The statistical results are based on 5000 Monte-Carlo experiments.

In the first simulation, we compare the performance of the proposed HAML technique used with 2 BSs to the original AML approach with 3 or more BSs. The MS position in $\mathrm{m}$ is at $(1000,2000)$. Table 1 lists the root mean squares (rms) of location error calculated as follows:

$$
\mathrm{rms}=\left(\frac{1}{5000} \sum_{k=1}^{5000}\left(\bar{x}_{k}-x^{0}\right)^{2}+\left(\bar{y}_{k}-y^{0}\right)^{2}\right)^{1 / 2},
$$

where the superscript ()$^{0}$ stands for the true value.

Although the AML technique uses a larger number of BSs, the HAML method shows better performance, as illustrated in Table 1. The accuracy of AML surpasses that of HAML only if 7 BSs are involved in the positioning process which may not necessarily be available in a real-world scenario.

In the second simulation, the mobile coordinates are varied on a linear trajectory, according to the expressions $x=1000+800 * x_{M S}$ and $y=1000+800 * y_{M S}$ where $x_{M S}$ and $y_{M S}$ are two random variables uniformly distributed in $[0,1]$. The rms error is averaged over 100 ensemble runs. Figure 1 illustrates cumulative distribution function (CDF) of the error. As shown in this figure although the number of used BSs with the HAML technique is less than that of the AML approach, the performance of HAML is better than AML by providing a gain in accuracy of about $13 \%$.

In the third simulation we study the influence of the variability of TOA variances between BSs, which is a real-world possibility in wireless cellular networks [6]. As shown in Figure 2, when we increase the difference in TOA variance between BSs, the AML performance becomes worse. However, the HAML is less influenced by the increase of this feature because of the contribution of the added AOA 


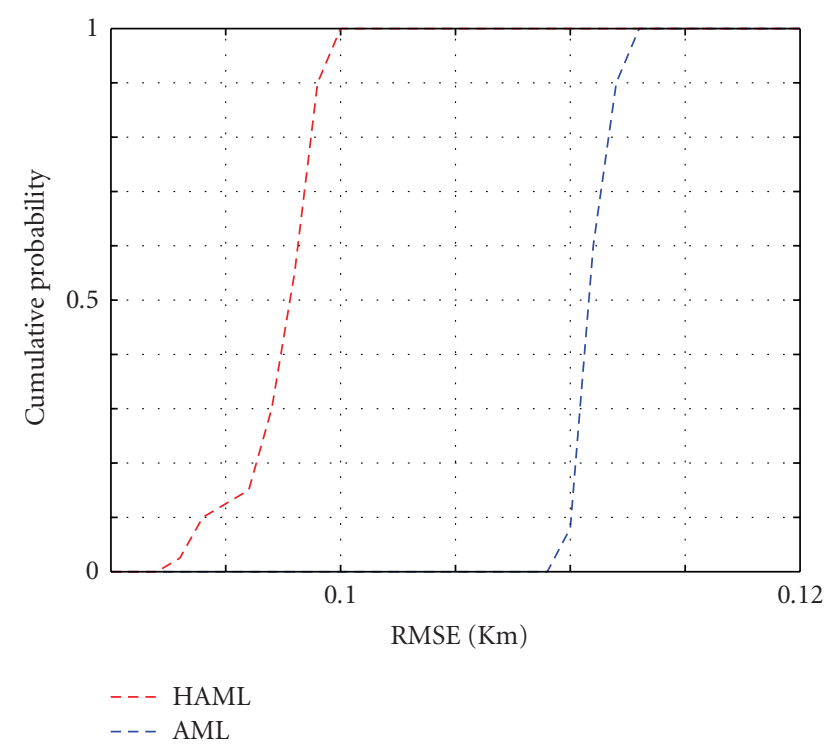

Figure 1: Comparison between 2-BS HAML and 3-BS AML techniques.

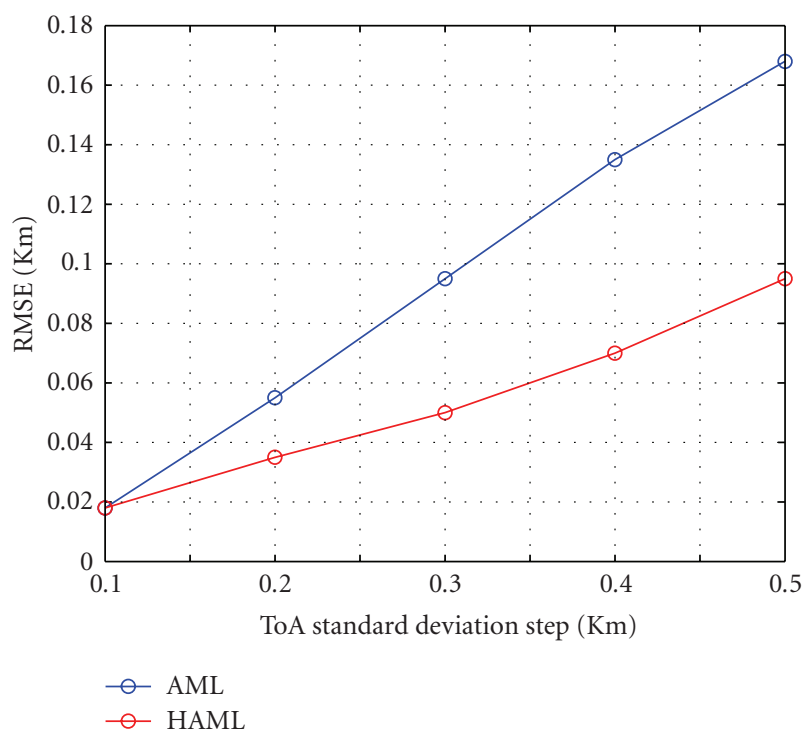

FIGURE 2: Influence of the variability of TOA variances between BSs on the position accuracy with 2-BS HAML and 3-BS AML techniques.

information, which in this case diminishes the influence of the TOA variances on the final accuracy by sharing the equations' weighting coefficients.

The last simulation compares the performance of AML and HAML techniques against the AOA standard deviation. As depicted in Figure 3, the accuracy of the mobile position estimate calculated with the HAML algorithm is enhanced when more accurate AOA measurements are used. However and as expected, no effect is introduced to the position estimate calculated with the AML technique since it does not use the AOA information. It is worth noticing that the

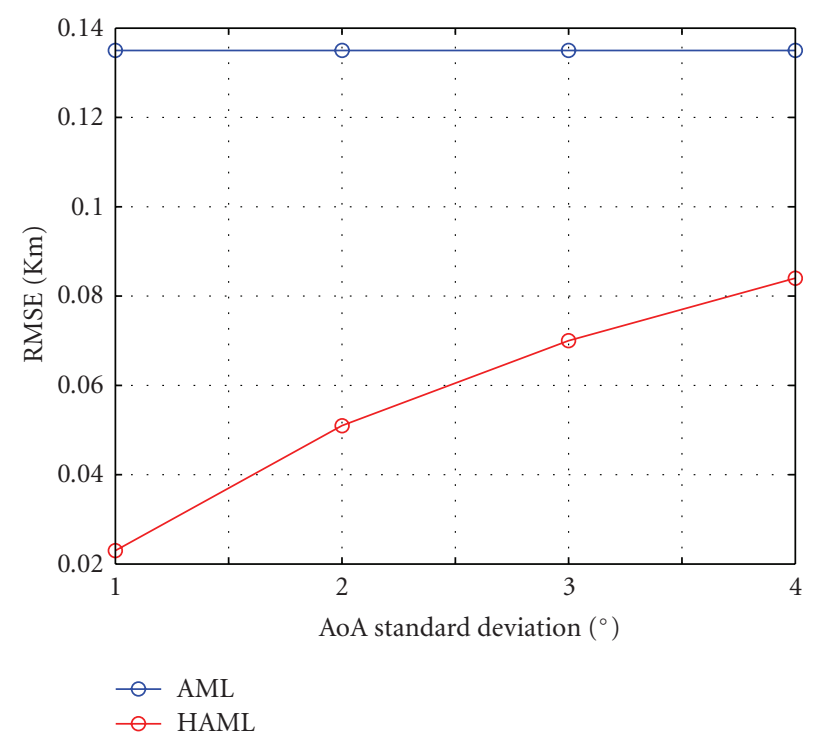

FIGURE 3: Influence of the AOA variances on the position accuracy with 2-BS HAML and 3-BS AML techniques.

performance of the proposed HAML algorithm still be better than that of the AML method using any AOA standard deviation.

\section{Conclusion}

In this letter a hybrid approximate maximum likelihood (HAML) approach for wireless location was proposed. The main contribution of this technique is that it can achieve good performance with TOA/AOA measurements from only two base stations, thereby avoiding problems of hearability and network overload. Simulation shows that the proposed technique is less influenced by the difference of noise powers between BSs. Moreover, in some geometric cases HAML results obtained from 2 BSs are as accurate as AML results obtained from 7 BSs.

\section{Acknowledgment}

Mohamed Adnan Landolsi is duly acknowledges the support of King Fahd University of Petroleum\& Minerals.

\section{References}

[1] C. Drane, M. Macnaughtan, and C. Scott, "Positioning GSM telephones," IEEE Communications Magazine, vol. 36, no. 4, pp. 46-54, 59, 1998.

[2] Y. Zhao, "Mobile phone location determination and its impact on intelligent transportation systems," IEEE Transactions on Intelligent Transportation Systems, vol. 1, no. 1, pp. 55-64, 2000.

[3] Y. T. Chan, H. Y. C. Hang, and P. C. Ching, "Exact and approximate maximum likelihood localization algorithms," IEEE Transactions on Vehicular Technology, vol. 55, no. 1, pp. 10-16, 2006. 
[4] J. J. Caffery Jr. and G. L. Stuber, "Overview of radiolocation in CDMA cellular systems," IEEE Communications Magazine, vol. 36, no. 4, pp. 38-45, 1998.

[5] S. M. Kay, Fundamentals of Statistical Signal Processing: Estimation Theory, Prentice-Hall PTR, Upper Saddle River, NJ, USA, 1993.

[6] M. A. Landolsi, A. H. Muqaibel, and A. S. Al-Ahmari, "Nearfar problem impact on mobile radiolocation accuracy in CDMA wireless cellular networks," in Proceedings of the IEEE International Conference on Telecommunications and Malaysia International Conference on Communications (ICT-MICC '07), pp. 14-17, Penang, Malaysia, May 2007. 

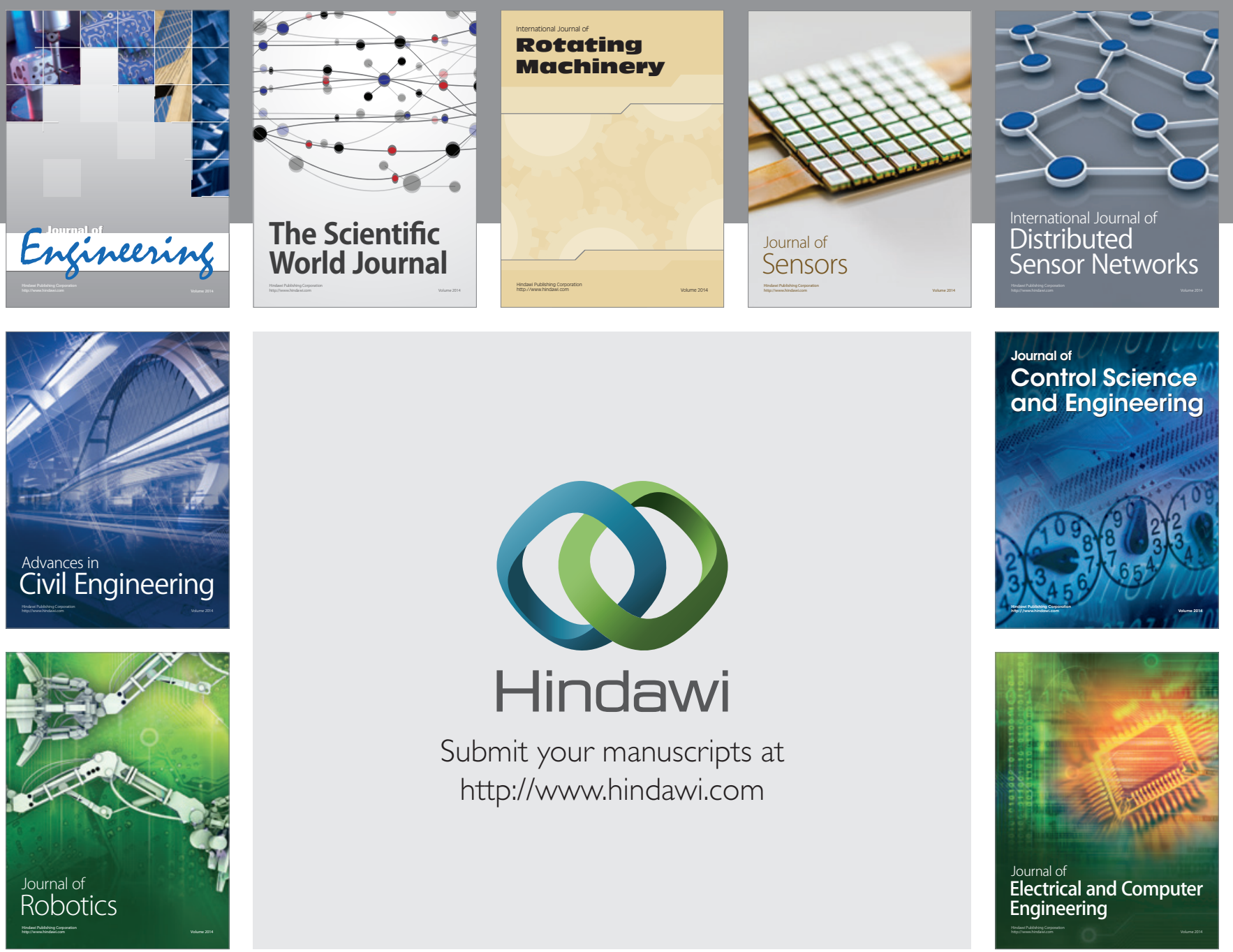

Submit your manuscripts at

http://www.hindawi.com
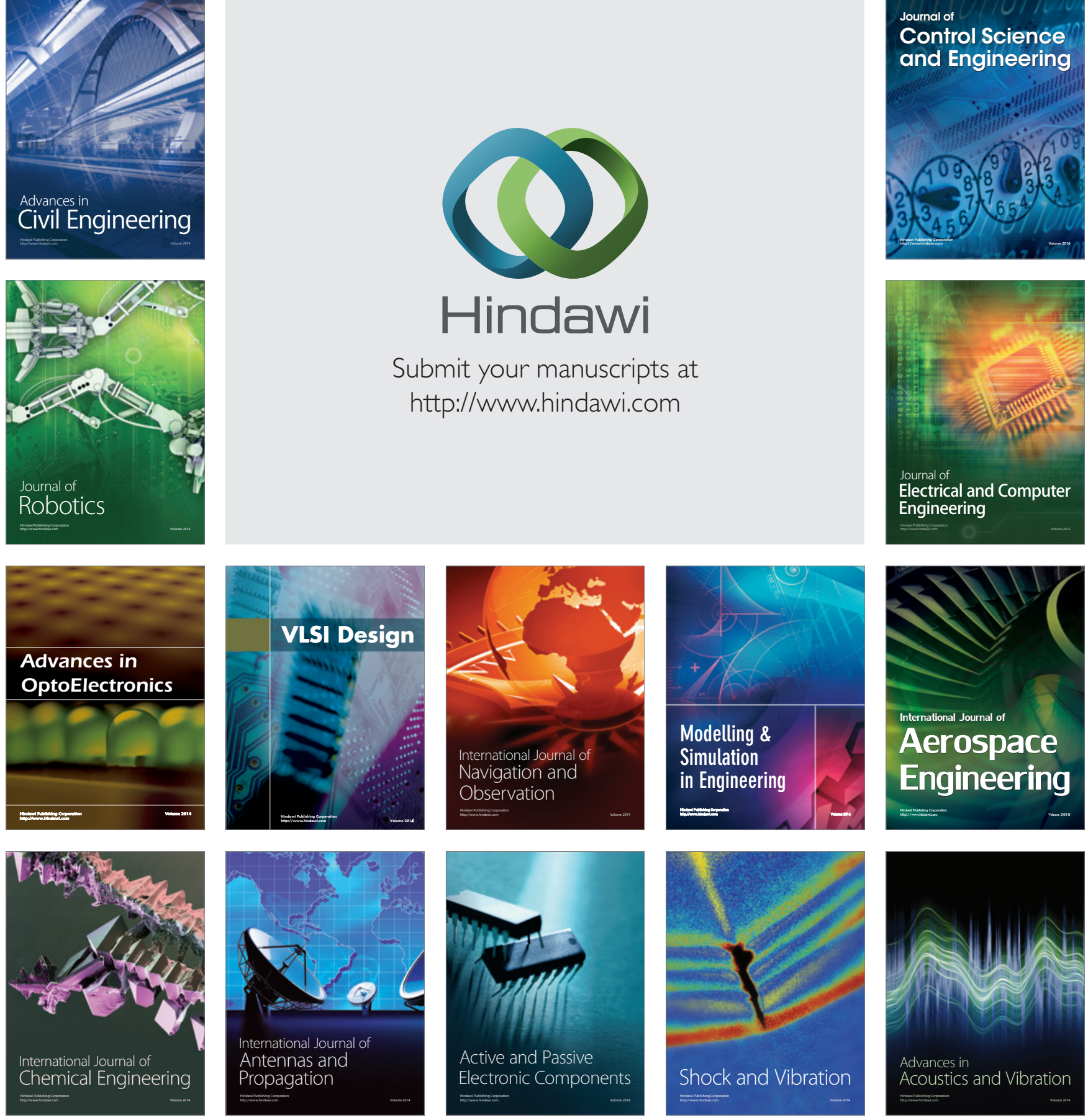